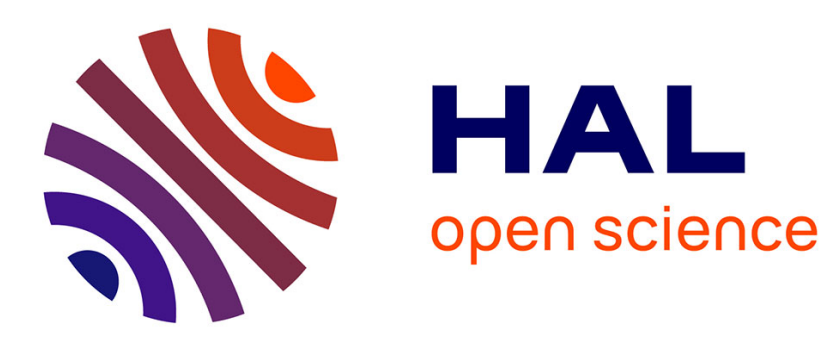

\title{
Extinction ratio enhancement by sinusoidal phase modulation and nonlinear spectral focusing
}

Frederic Audo, Christophe Finot

\section{To cite this version:}

Frederic Audo, Christophe Finot. Extinction ratio enhancement by sinusoidal phase modulation and nonlinear spectral focusing. Laser Physics, 2017, 27, pp.115401. 10.1088/1555-6611/aa8291 . hal01552203

\section{HAL Id: hal-01552203 \\ https://hal.science/hal-01552203}

Submitted on 1 Jul 2017

HAL is a multi-disciplinary open access archive for the deposit and dissemination of scientific research documents, whether they are published or not. The documents may come from teaching and research institutions in France or abroad, or from public or private research centers.
L'archive ouverte pluridisciplinaire HAL, est destinée au dépôt et à la diffusion de documents scientifiques de niveau recherche, publiés ou non, émanant des établissements d'enseignement et de recherche français ou étrangers, des laboratoires publics ou privés. 


\title{
Extinction ratio enhancement by sinusoidal phase modulation and nonlinear spectral focusing
}

\author{
F Audo and C Finot \\ Laboratoire Interdisciplinaire Carnot de Bourgogne, UMR 6303 CNRS - Université de \\ Bourgogne-Franche-Comté, 9 avenue Alain Savary, BP 47870, 21078 Dijon Cedex, \\ France \\ E-mail: christophe.finot@u-bourgogne.fr
}

\begin{abstract}
We propose a new approach to enhance the extinction ratio of a pulse impaired by a coherent continuous-wave background. Taking advantage of a sinusoidal phase modulation followed by a nonlinear spectral focusing and an optical bandpass filtering, we are able to significantly decrease the level of the unwanted background. The principle of the method is experimentally validated at telecommunication wavelengths.
\end{abstract}

Keywords. Signal processing, spectral compression, nonlinear propagation.

\section{Introduction}

Optical pulses with durations of tens or hundreds of picoseconds are required for a wide range of applications. An efficient cavity-free solution to generate such pulses is to use a continuous wave laser that is externally modulated in the temporal domain by an optical modulator driven by a high-bandwidth electrical waveform generator. However, the extinction ratio of these modulators is finite and is usually limited to around twenty $\mathrm{dBs}$. As a direct consequence, for pulse trains with a low duty cycle, the overall energy remaining in the residual coherent continuous background is not negligible and can be of the same order as the energy contained within the pulse. This may therefore seriously impair the practical use of the pulse train by creating unwanted interferences or by affecting the efficiency of subsequent amplification stages.

In this context, it becomes of high interest to develop all-optical approaches to remove this spurious background. Many solutions have been proposed in the field of optical communications using dedicated components such as saturable absorbers [1,2], or artificial absorbers taking advantage of the nonlinear effects occurring in optical fibers. Indeed, nonlinear optical loop mirrors [3, 4], Mamyshev's regenerators $[5,6]$, spectral-compression based devices [7] or four-wave mixing processes [8] present a nonlinear 
relationship between the input and output powers, enabling the enhancement by several $\mathrm{dB}$ of the extinction ratio.

We propose here a new method based on the use of a sinusoidal phase modulation followed by nonlinear spectral focusing and optical bandpass filtering. After having described the principle of our approach, we experimentally validate its efficiency by demonstrating a reduction by more than $15 \mathrm{~dB}$ of the continuous background.

\section{Principle of operation}

In order to illustrate our approach, let us consider a chirp-free Gaussian pulse with a temporal profile $G(t)$ lying on a continuous background with a level that is $E R=23 \mathrm{~dB}$ lower than the peak power $P_{0}$ of the pulse: $\psi_{0}(t) \simeq \sqrt{P_{0}}(G(t)+\sqrt{E R})$. Our approach can be applied to other pulse waveforms as long as their temporal intensity profile in the central part can be approximated to a good extend by a parabolic fit [9]. We consider here parameters that are typical of the experimental validation that will be described in section 3 and 4 . The initial pulses have a 120 ps full-width at half-maximum (FWHM) pulse duration and a repetition rate of $78.13 \mathrm{MHz}$, leading to a duty-cycle of $0.94 \%$. The spectral intensity profile corresponding to $\psi_{0}(\omega) \simeq G(\omega)+\sqrt{E R} \delta(\omega)$ is plotted on figure 1(b1) where we can make out in the spectral domain that the Gaussian spectrum linked to the pulsed part is more than $21 \mathrm{~dB}$ below the level of the continuous wave.

The initial signal is then externally phase modulated by a temporal sinusoidal waveform $\varphi(t)=\beta \cos \left(\omega_{\text {mod }} \cdot t+\phi_{0}\right)$ with an angular frequency $\omega_{\text {mod }}$, an amplitude $A$ and a phase offset $\phi_{0}$. The resulting spectrum is made of replicas of the initial spectrum spaced by $\omega_{\bmod }[10]$ :

$$
\begin{gathered}
\psi_{\text {mod }}(\omega)=\psi_{0}(\omega) *\left(\sum_{n=-\infty}^{\infty} J_{n}(\beta) \delta\left(\omega-n \omega_{\mathrm{mod}}\right)\right) \\
\psi_{\mathrm{mod}}(\omega)=\sum_{n=-\infty}^{\infty} J_{n}(\beta)\left(G\left(\omega-n \omega_{\mathrm{mod}}\right)+\sqrt{E R} \delta\left(\omega-n \omega_{\mathrm{mod}}\right)\right)
\end{gathered}
$$

where $J_{n}$ is the Bessel function of the first kind of the $n$-th order. The amplitude of the phase modulation is chosen to be $\beta=2.405$ so that the component centered at $\omega=0$ vanishes, i.e. $J_{0}(\beta)=0$. Therefore, as can be seen in panel b2 of figure 1, the central coherent line has disappeared and its energy is now spread into a set of equally spaced spectral lines. Note that other periodic phase modulation schemes may also lead to spectrum splitting into lateral bands [11], however, they will not be suitable for the second stage of operation.

After the external phase modulation that induces a sinusoidal chirp in the temporal domain, the signal is propagated in a Kerr medium such as a highly nonlinear fiber that induces self-phase modulation (SPM). The resulting chirp is directly linked to the temporal gradient of the initial intensity profile $\left|\psi_{0}(t)\right|^{2}$ and is therefore maximum for the pulsed part $|G(t)|^{2}$ and null for the coherent background. If usually SPM is associated with spectral expansion and the generation of new frequencies in the spectrum $[12,13]$, this is not always the case when the input wave is already chirped with a chirp slope opposite to the SPM chirp. In this context, a spectral redistribution of the components occurs, leading to a spectral narrowing of the pulse $[12,14,15]$. Usually, the input chirp required to observe spectral compression is imparted by an anomalously dispersive element, but it can also be achieved through external temporal phase sinusoidal modulation, provided $\phi_{0}=\pi$. Given the temporal duration of the pulse under study, we 
can consider that the dispersive effects can be neglected [16] and assume a perfectly nonlinear propagation where the SPM impact is limited to an additional temporal phase term $\gamma \cdot|G(\mathrm{t})|^{2} \cdot L$, with $L$ being the length of the fiber and $\gamma$ its nonlinear coefficient. Inspired by our previous works [17, 18], we have carefully chosen the $\omega_{\text {mod }}$ in order to observe an optimal compensation of the SPM-induced chirp by the sinusoidal phase modulation $\left(\omega_{\text {mod }}=4.45 \mathrm{GHz}\right)$. Therefore, for an input peak power of $1.15 \mathrm{~W}$, we can make out in panel (a1) that the chirp is very significantly reduced over most of the pulse extend. This leads to an efficient narrowing, as can be seen in panels (b3) where nearly $95 \%$ of the energy of the pulsed signal has gone back around the central wavelength.
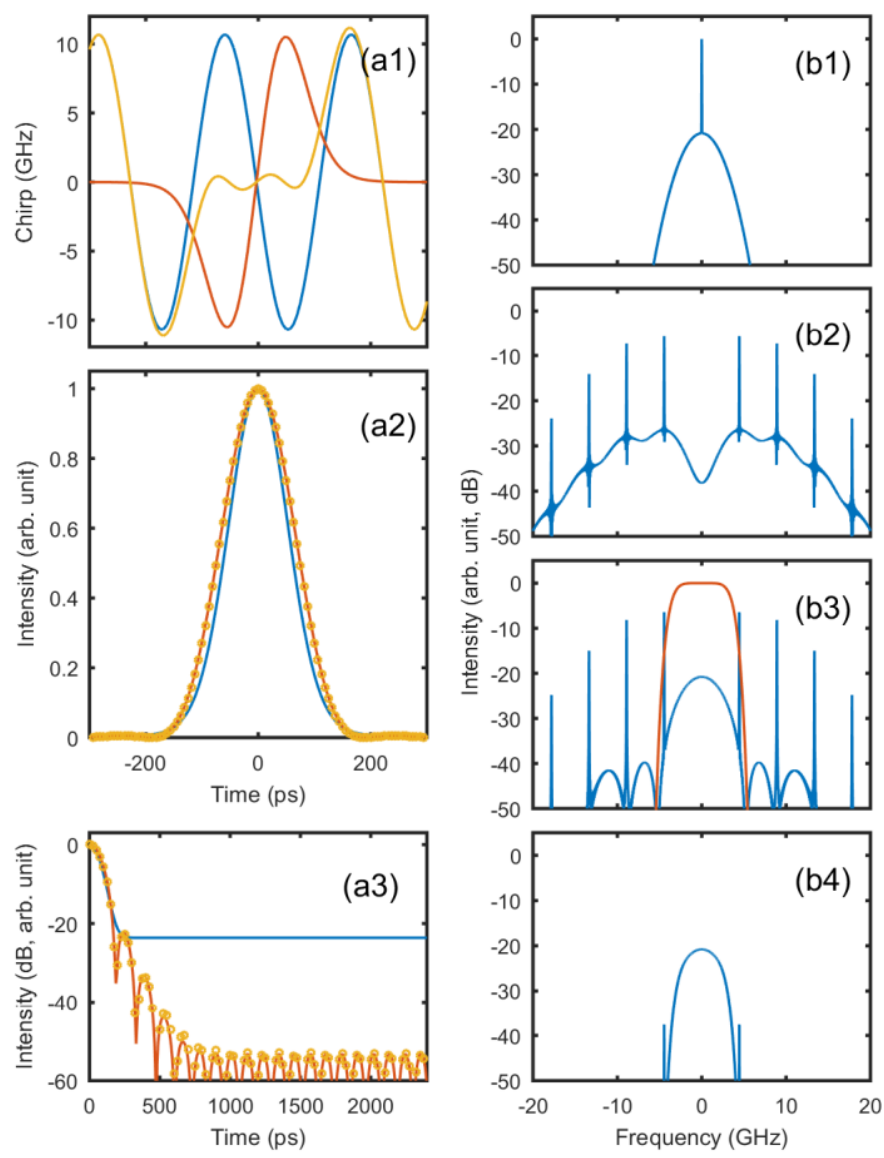

Figure 1 - Evolution of the temporal and spectral properties of the signal (subplots a and b, respectively). The initial temporal and spectral intensity profiles are provided in panels a2 (blue line) and b1. Panel b2 shows the spectrum obtained after phase modulation whereas the blue curve of panel a1 stands for the chirp induced by phase modulation in the temporal domain. The chirp induced by SPM of the Gaussian pulse is plotted with red curve in panel a1 and the resulting chirp of the signal after nonlinear propagation is plotted in yellow curve. Plot b3 shows the resulting spectral intensity profile (blue line) after spectral focusing as well as the filter under use (red curve). Panel b4 represents the spectral profile after filtering. The corresponding intensity profile is plotted in yellow in panel a2 and is compared with the profile assuming a Fourier-limited pulse (circle). Panel a3 shows the input and filtered intensity profiles on a logarithmic scale (red and blue curves respectively).

As the SPM only affects the pulsed part of the signal, the coherent background remains unchanged and no energy from the residual wave is focused back to the center of the spectrum. Therefore, an optical bandpass filter (OBPF) centered at the central wavelength may easily remove the coherent background. 
We can clearly observe on the panel b4 that the signature of the coherent wave has nearly completely disappeared. In the temporal domain, the level of the coherent background has been decreased by more than $20 \mathrm{~dB}$ (see inset a3). The filtered pulse is extremely close from the Fourier transform limit, indicating that the chirp has been nearly canceled. However, the temporal intensity profile is slightly broadened compared to the initial one. The sharp edge filter under use leads to rapidly decaying oscillations in the temporal profiles as can be noticed when plotted on a logarithmic scale.

Let us note here that self-phase modulation combined with optical bandpass filtering has already been involved in the past for optical regeneration in a device known as Mamyshev regenerator [5, 6]: the offset frequency filtering of the spectrally broadened spectrum can achieve an efficient suppression of the continuous wave. However, contrary to our proposed method, such a scheme imposes a wavelength conversion of the signal and requires higher peak power. Much more recently [7], spectral focusing followed by optical bandpass filtering has also been suggested to increase the signal-to-noise ratio and to reduce the amplitude jitter of a data pulse stream. However, in that work, the pulses obtained after processing have experienced significant changes of their temporal and spectral properties with for example a temporal stretching by a factor above 10 .

\section{Experimental setup}

The all-fibered experimental setup is sketched in figure 2 and relies exclusively on commercially available components from the telecom industry. The initial continuous wave is delivered by an external cavity laser at $1550 \mathrm{~nm}$ and is intensity modulated by a Lithium-Niobate modulator with a bandwidth of $20 \mathrm{GHz}$. The electrical signal that drives the modulator is the result of the electrical filtering by a $5.4 \mathrm{GHz}$ low-pass filter of an on-off keyed signal from a $10 \mathrm{GHz}$ pattern generator. As a 128-bit sequence with 1 one followed by 127 zeros is chosen, a repetition rate of $78.13 \mathrm{MHz}$ is obtained. A second modulator is driven by a $4.69 \mathrm{GHz}$ electrical clock synchronized with the first electrical signal. It imprints a sinusoidal phase on the optical wave with the appropriate phase offset $\phi_{0}=\pi$ and amplitude $\beta=2.405$. The resulting signal is then amplified by an erbium doped fiber amplifier and sent into a $500 \mathrm{~m}$ highly nonlinear fiber

with $\gamma=10 \mathrm{~W}^{-1} / \mathrm{km}^{-1}$. The linear attenuation of the fiber is $0.7 \mathrm{~dB} / \mathrm{km}$ and the dispersion of the fiber is normal $(D=-1 \mathrm{ps} / \mathrm{km} / \mathrm{nm})$ in order to prevent from any degradation that may be linked to modulation instability. A narrow bandwidth OBPF with a close to rectangular shape is finally used to remove the unwanted sidebands from the processed signal.

The optical signal is characterized at different stages both in the temporal and spectral domains by a $40 \mathrm{GHz}$ electrical sampling oscilloscope and a high resolution optical spectrum analyzer $(100 \mathrm{MHz}$ resolution, APEX 2443B). Let us remark that the order of the external phase-modulation and the SPM stages can be modified as long as dispersion does not play any significant role in the propagation. 


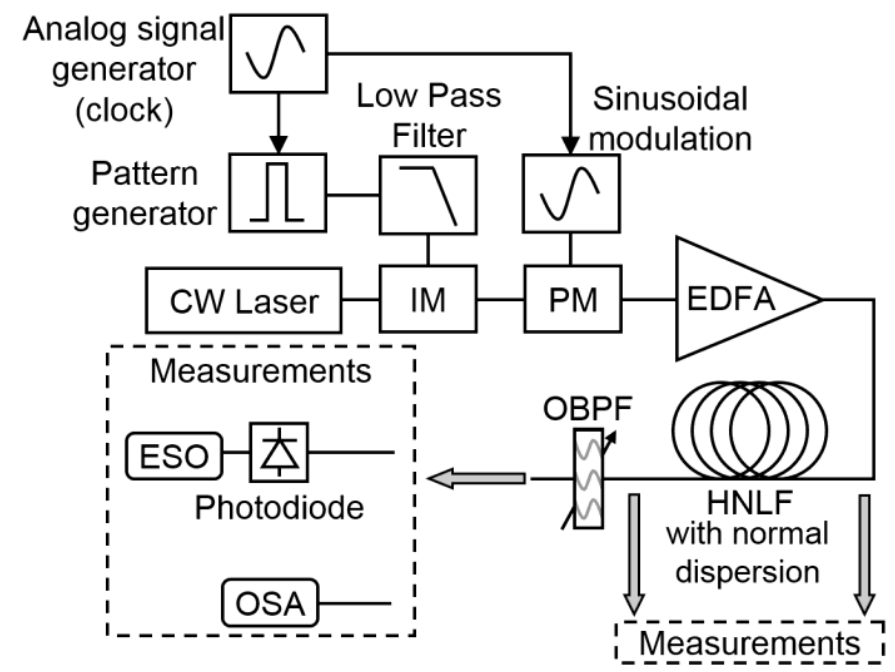

Figure 2 - Experimental setup. IM: Intensity Modulator ; PM: Phase Modulator ; EDFA: Erbium Doped Fiber Amplifier ; HNLF: Highly Nonlinear Fiber ; OSA: Optical Spectrum Analyzer ; ESO: Electrical Sampling Oscilloscope ; OBPF: Optical Bandpass Filter.

\section{Experimental results}

Temporal and spectral properties of the pulse at different stages of the setup are summarized in figure 3 . The initial pulse obtained after optimization of the working parameters of the intensity modulator (especially the bias voltage in order to reduce as much as possible the background) has a FWHM pulse duration of $120 \mathrm{ps,} \mathrm{leading} \mathrm{to} \mathrm{a} \mathrm{duty} \mathrm{cycle}$ of $0.94 \%$. Let us note that the noise performance of our temporal detection does not allow us to determine the exact level of the background. Given the large continuous component visible in the initial spectrum (panel b1), the energy contained in the coherent background is far from being negligible. From the spectral measurements, we estimate than $35 \%$ of the energy is contained within the central coherent component, leading to an extinction ratio of $23 \mathrm{~dB}$, which is fully consistent with the technical datasheet of the commercial intensity modulator. 

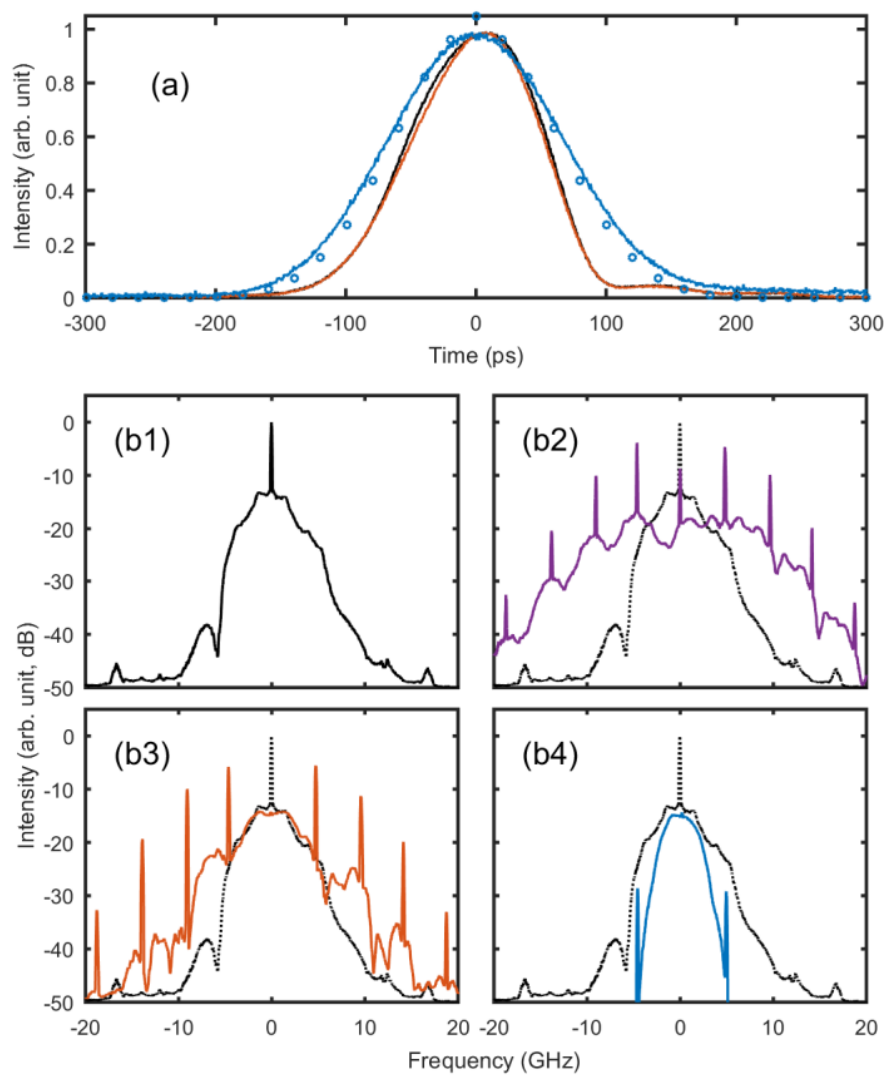

Figure 3 - Temporal and spectral intensity profiles of the pulse train (panels a and b respectively). Spectral intensity profiles obtained directly after intensity modulation, after phase modulation, after nonlinear propagation and after spectral filtering are plotted with solid lines in panels b1, b2, b3 and b4, respectively. The dotted line in panels b(2-4) is for the input spectrum. The circles used in panel 4a refers to the intensity profile that can be inferred from the optical spectrum in the case of a pulse in the Fourier transform limit.

The power driving the phase modulator is adjusted in order to be as close as possible from the point where the central continuous harmonics vanishes. For this amplitude of phase modulation, the energy is transferred to higher-order sidebands as shown in panel b2. The nonlinear propagation within the hnlf enables the spectral focusing of the energy associated to the pulse. The injected optical power in the hnlf is optimized to observe the optimum spectral narrowing of the pulsed part. We observe in panel b3 that experimentally the spectral compression stage does not affect the lateral coherent sidebands created by the phase modulation. The temporal intensity profiles are not affected neither by the phase modulation nor by the spectral compression.

The final stage is to take advantage of a narrow bandwidth filter with sharp edges to isolate the central part of the spectrum and to eliminate the sidebands associated to the continuous background. The resulting spectral profile is given in panel b4 that highlights that the signatures of the continuous background have been dramatically reduced. From the output spectrum, we estimate that the energy in the coherent background now represents only $0.5 \%$ of the total energy of the signal, leading to an enhancement of $20 \mathrm{~dB}$ of the extinction ratio. Let us however note that as our setup involves an amplification stage in order to reach the adequate peak power, some amplified spontaneous emission may slightly degrade the intrapulse OSNR. The use of an optical filter with sharp edges limits this unwanted ASE. The temporal intensity profile does not show any deleterious degradation linked to the filtering 
process. On the contrary, a small ripple visible in the trailing edge of the initial pulse has been removed in the process. We can finally compare the recorded intensity profile with the one expected from the optical spectrum when assuming a Fourier transform profile: the temporal durations and shapes of the temporal profiles are rather similar, indicating that the final pulse obtained experimental is reasonably close from the Fourier-transform limit.

\section{Conclusion}

We have presented a new, simple approach to enhance the extinction ratio of a pulse train affected by a coherent residual background. Combining a sinusoidal phase modulation with nonlinear spectral focusing and spectral filtering, we have theoretically and experimentally demonstrated the possibility to enhance by more than $10 \mathrm{~dB}$ the extinction ratio of the signal. With phase modulators sustaining operation up to $40 \mathrm{GHz}$ and low-dispersion highly nonlinear fibers, our approach can be adapted to pulse durations as short as 15 picoseconds. The scheme can also be combined during the nonlinear propagation with distributed amplification [18]. Given the low level of SPM that is required, the process is suitable for onchip operation [19-21]. We have experimentally demonstrated the improvement of signal degraded by the finite modulation depth of a Lithium-Niobate Modulator. Our approach could also work with others cavity-free pulse generation schemes method such as the one based on the parametric amplification of a frequency shifted continuous wave [22]. Given the space-time duality [23], we may also extend our approach to the cleaning of a residual coherent background of a spatial beam.

\section{Acknowledgements}

The article has benefited from the PICASSO experimental platform of the University of Burgundy. We thank Sonia Boscolo for unvaluable discussions on nonlinear spectral focusing. The authors would like to acknowledge support by the Région Bourgogne (Pari Photcom) and the Agence Nationale de la Recherche (Labex Action ANR-11-LABX-01-01). This work was supported by the European Union within the framework of the operational Program FEDER-FSE Bourgogne 2014-2020 and by the Institut Universitaire de France.

\section{References}

1. Massoudre D, Oudar JL, Fatome J, Pitois S, Millot G, Decobert J, et al. 2006 Opt Lett 31 537-9

2. Gay M, Costa e Silva M, Nguyen TN, Bramerie L, Chartier T, Joindot M, et al. 2010 IEEE Photon Technol Lett 22 158-60

3. $\quad$ Doran NJ, Wood D 1988 Opt Lett 13 56-8

4. Boscolo S, Turitsyn SK, Blow KJ 2008 Opt Fiber Technol 14 299-316

5. Mamyshev PV, editor. All-optical data regeneration based on self-phase modulation effect. European Conference on Optical Communication, ECOC'98; 1998; Institute of Electrical and Electronics Engineering, Madrid, Spain.

6. Provost L, Finot C, Mukasa K, Petropoulos P, Richardson DJ 2007 Opt Express 15 5100-13

7. $\quad$ Boscolo S, Fatome J, Finot C 2017 Opt Commun 389 197-202

8. Ciaramella E, Curti F, Trillo S 2001 IEEE Photon Technol Lett 13 142-4

9. Boscolo S, Chaussard F, Andresen ER, Rigneault H, Finot C 2017 submitted preprint available https://hal.archives-ouvertes.fr/hal-01502006

10. Goodman JW. Introduction to Fourier Optics: Roberts \& Co; 2005.

11. Finot C, Rigneault H 2017 preprint available https://hal.archives-ouvertes.fr/hal-01501999 
12. Stolen RH, Lin C 1978 Phys Rev A 17 1448-53

13. Agrawal GP. Nonlinear Fiber Optics, Fourth Edition. San Francisco, CA: Academic Press; 2006.

14. Zohrabian AV, Mouradian LK 1995 Quantum Electron 251076

15. Oberthaler M, Höpfel RA 1993 Appl Phys Lett 63 1017-9

16. Finot C, Boscolo S 2016 J Opt Soc Am B 33 760-7

17. Boscolo S, Mouradian LK, Finot C 2016 J Opt 18105504

18. Audo F, Boscolo S, Fatome J, Kibler B, Finot C 2017 submitted for publication preprint available https://hal.archives-ouvertes.fr/hal-01537838v1

19. Madden SJ, Choi DY, Bulla DA, Rode AV, Luther-Davies B, Ta'eed VG, et al. 2007 Opt Express 15 14414-21

20. Levy JS, Saha K, Okawachi Y, Foster MA, Gaeta AL, Lipson M 2012 IEEE Photon Technol Lett 24 1375-7

21. Kuyken B, Ji H, Clemmen S, Selvaraja SK, Hu H, Pu M, et al. 2011 Opt Express 19 B146-B53

22. Wiberg AOG, Bres CS, Kuo BPP, Zhao JX, Alic N, Radic S 2009 IEEE J Quantum Electron 45 $1325-9$

23. Kolner BH 1994 IEEE J Quantum Electron 30 1951-63 